\title{
The strengths and limitations of routine staging before treatment with abdominal CT in colorectal cancer
}

Irene Grossmann ${ }^{1,2^{*}}$, Joost M Klaase ${ }^{2}$, Johannes KA Avenarius ${ }^{3}$, Ignace HJT de Hingh ${ }^{4}$, Walter JB Mastboom² and Theo Wiggers ${ }^{1}$

\begin{abstract}
Background: Advanced colorectal cancer (CRC), either locally advanced, metastasized (mCRC) or both, is present in a relevant proportion of patients. The chances on curation of advanced CRC are continuously improving with modern multi-modality treatment options. For incurable CRC the focus lies on palliation of symptoms, which is not necessarily a resection of the primary tumor. Both situations motivate adequate staging before treatment in CRC. This prospective observational study evaluates the outcomes after the introduction of routine staging with abdominal $\mathrm{CT}$ before treatment.
\end{abstract}

Methods: In a prospective observational study of 612 consecutive patients (2007-2009), the ability of abdominal CT to find liver metastases (LM), peritoneal carcinomatosis (PC) and T4 stage in colon cancer (CC) was analysed.

Results: Advanced CRC was present in 58\% of patients, mCRC in 31\%. The ability to find LM was excellent (99\%), CT4 stage CC good (86\%) and PC poor (33\%). In the group of surgical patients with emergency presentations, the incidences of both mCRC (51\%) and locally advanced colon cancer (LACC) (69\%) were higher than in the elective group (20\% and $26 \%$ respectively). Staging tended to be omitted more often in the emergency group (35\% versus $12 \%$ in elective surgery).

Conclusions: The strengths of staging with abdominal CT are to find LM and LACC, however it fails in diagnosing PC. On grounds of the incidence of advanced CRC, staging is warranted in patients with emergency presentations as well.

\section{Background}

Advanced colorectal carcinoma (CRC), defined as locally advanced or metastasized disease or both, is present in a relevant proportion of patients diagnosed with colorectal cancer. Common localizations for distant metastases are the liver, the peritoneal cavity and the lung. Staging with chest $\mathrm{CT}$ as a routine procedure before surgery has not shown to be of clinical benefit, mainly due to the low incidence of clinically relevant lung metastases and low specificity of chest CT [1-3]. Pre-operative staging with abdominal CT might be beneficial when the ability to detect advanced CRC is high enough and the findings

\footnotetext{
* Correspondence: irenegrossmann@me.com

'Department of Surgery, University Medical Center Groningen, (Hanzeplein

1), Groningen, (9713 GZ), the Netherlands

Full list of author information is available at the end of the article
}

offer information that may change the treatment plan. Such findings include liver metastases (LM), peritoneal carcinomatosis (PC) and locally advanced colon cancer (LACC). In the past, these conditions were frequently regarded as incurable and suitable for palliative measures only. Nowadays various multi-modality treatments offer a chance of cure to selected patients [4-15]. For patients with incurable advanced CRC, alternative options are sometimes available and staging may change the treatment plan towards the 'best palliative care', that may consist of avoidance of surgery in selected patients [16-18]. At the present, adequate staging with CT before treatment is already considered as the standard in international CRC guidelines. However the evidence for this advice in literature is mostly limited to studies focused on the radiological accuracy and also are usually
C Biomed Central 
conducted in selected patient groups. The actual findings of staging after implementation of such guideline has not been described before. An observational study may be helpful in the debate on actual clinical relevance and provide lead points to optimize staging in clinical practice.

The aim of this study is to describe the outcomes of routine staging with abdominal $\mathrm{CT}$ in a unselected hospital population $(n=612)$ after the introduction of a regional guideline recommendation in 2007.

\section{Methods}

The data were collected in the Medical Spectrum Twente, a large community teaching hospital in the regional capital of a foremost rural area in the eastern part of the Netherlands. It functions as a regional referral center for liver and lung surgery, but has no facilities for the treatment of peritoneal carcinomatosis with hyperthermic intraperitoneal chemotherapy (HIPEC).

The study design is a prospective observational cohort study evaluating the outcome of routine staging with abdominal CT concerning the ability to find liver metastases (LM), peritoneal carcinomatosis (PC) and T4-stage in colon cancer (LACC). All patients in our hospital who where treated for CRC from January 2007 till December 2009 were included in the analysis; all surgical patients with CRC in the study hospital were prospectively registered in a database designed for colorectal surgery, including patient characteristics, staging and surgical procedures, the clinical $M$ stage and pathological TNM stage, post-operative mortality, treatment of metastases and follow-up; patients with the diagnosis of CRC in the same 3 years who did not undergo surgery were identified by the regional cancer registry and retrospectively added to the database. The clinical T stage of colon cancer on abdominal CT (cT4 or non-cT4) was retrospectively scored based upon the original radiology reports.

Routine pre-operative staging with a CT of chest and abdomen for patients with CRC was introduced as a regional CRC guideline in 2007 and preceded similar national guideline recommendations (2008). CT scanning was performed on a 16 and 64 slice scanner (Toshiba Aquillion 16 and 64) after intravenous contrast injection (visipaque $320,90 \mathrm{ml}, 3 \mathrm{ml} / \mathrm{s}$.) in the portal venous phase, with a slice thickness of $1 \mathrm{~mm}$ and a reconstruction of $0.8 \mathrm{~mm}$. When preoperative scanning was omitted, staging with abdominal $\mathrm{CT}$ was intended within 3 months after surgery. Patients with rectal cancer, defined as localization below the peritoneal reflection, were additionally staged with a pelvic MRI for determination of the local invasion and possible lymph node metastases (cTN stage) and received neo-adjuvant (chemo)radiation according to the Dutch guidelines on rectal cancer. Follow-up after curative treatment of nonmetastatic CRC consisted of serum CEA measurements every 3 months combined with bi-annual ultrasound of the liver.

Pathological staging was based upon the TNM classification 2002 (6th edition) and classified according to the American Joint Committee on Cancer (AJCC) stages. Advanced CRC was defined as either locally advanced disease, presence of distant metastases or both. Locally advanced colon cancer (LACC) was defined by pT4 stage; meaning the tumor showed invasion through the serosal layer or into surrounding organs. Locally advanced rectal cancer (LARC) was defined as all patients that had either a T4 tumor or a T3 tumor with a threatened circumferential margin on pelvic MRI. The final diagnosis of liver metastases was based upon radiological (CT, contrast-enhanced ultrasound and/or PET scanning) and per-operative findings. In case of resection or in case of persistent uncertainty, histological confirmation was obtained. The final diagnosis of peritoneal carcinomatosis was by histological confirmation. The final diagnosis of lung lesions was on radiological grounds (chest CT). In case of resection or indeterminate lung lesions, histological confirmation was obtained when feasible. Several lung lesions remained indeterminate: these were followed by repeat CT scanning and considered positive when growth was observed [3]. Incurable CRC was defined as all macroscopical irradical (R2) resections of the primary tumor, when the patient had no resection of the primary tumor, or when no intended curative treatment of distant metastases was done.

Emergency presentation in the surgical patients was defined as all non-planned admissions to the hospital due to symptoms related to the tumor, with a subdivision into 'urgent' defined as surgery imperative within 5 days and 'acute' procedures within 6 hours.

The ability of the staging abdominal CT to detect advanced disease was analyzed in surgical patients that were staged with CT before treatment; the gold standard for PC and LACC were per-operative findings confirmed with histology. For LM the findings on CT (negative for liver metastases or indeterminate lesions) were related to per-operative findings and follow-up.

\section{Results}

In a 3 year period (2007-2009), 612 patients were diagnosed with colorectal carcinoma in the study center. Staging with abdominal CT before treatment was done in 513 patients $(84 \%)$. Surgery, either palliative or curative, was performed in 551 patients (90\%). The proportion of surgical patients with an emergency presentation was $21 \%(115 / 551)$. 
Population incidences of advanced colorectal cancer (Figure 1 and Table 1)

Metastatic CRC (mCRC) was diagnosed in 188 patients (31\%). Most common sites of distant metastases were the liver ( $n=144,24 \%)$, peritoneal cavity $(n=49,8 \%)$ and the lung $(n=42,7 \%)$. Intended curative treatment of metastases was feasible in $15 \%$ of patients with $\mathrm{mCRC}(\mathrm{n}=28)$. Locally advanced disease was present in 262 patients (43\%); on the total CRC population the incidence of LARC was $13 \%(n=81)$ and of LACC was
$30 \%(\mathrm{n}=181)$. Incurable disease was present in 188 patients $(31 \%)$, mainly due to $\mathrm{mCRC}(\mathrm{n}=160)$.

\section{Finding $\mathrm{mCRC}$ and/or LACC on abdominal CT (Table 2)}

The cohort of surgical patients that were staged before treatment $(n=463)$ was analyzed on the ability of the abdominal CT to find advanced disease. In this group, 86 patients had liver metastases; in 73 patients $(85 \%)$ diagnosed and in 19 patients (14\%) suspected (indeterminate lesions) on the initial staging CT. The

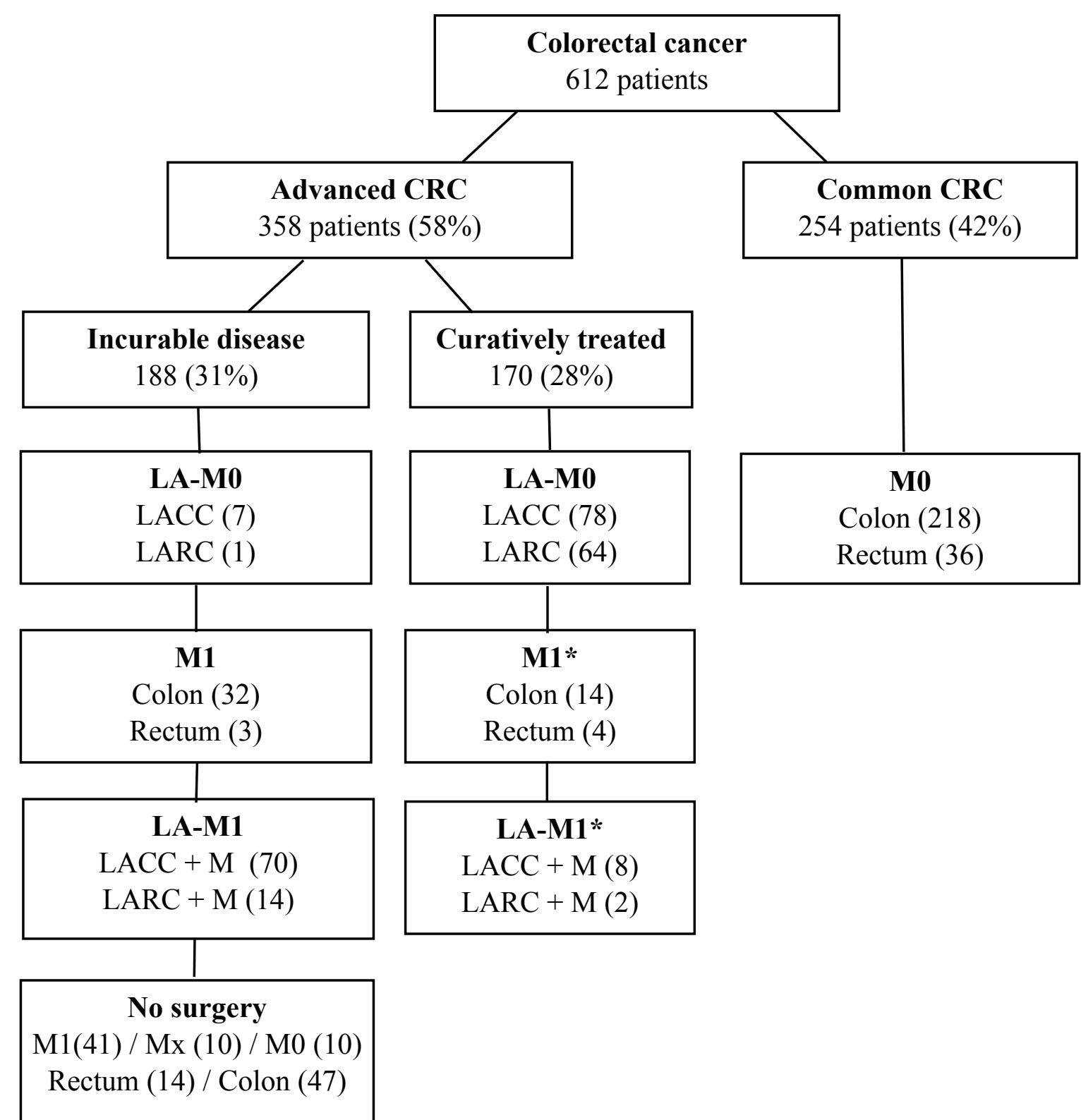

Figure 1 Colorectal cancer stage and treatment entire cohort. LA: locally advanced tumors (clinical on staging and/or pathological). M1: metastastic CRC. MO: no regional or distant metastases. LACC: locally advanced colon carcinoma = pT4 according to TNM 2002 (6th edition). LARC: locally advanced rectum carcinoma $=$ T4 tumors and T3 tumor with threatened circumferential margin on MRI. * ${ }^{*}$ Curative treatment distant metastases in 28 patients: liver 24, peritoneal 2, lung 2. 
Table 1 Characteristics all patients with CRC 2007-2009

\begin{tabular}{|c|c|c|}
\hline & \multicolumn{2}{|c|}{ All patients $(n=612)$} \\
\hline \multicolumn{3}{|l|}{ Age } \\
\hline Mean & $70 \mathrm{yr}$ & \\
\hline Median & $70 \mathrm{yr}$ & \\
\hline Range & $33-98$ & \\
\hline \multicolumn{3}{|l|}{ Gender } \\
\hline Male & 349 & $57 \%$ \\
\hline Female & 263 & $43 \%$ \\
\hline
\end{tabular}

\begin{tabular}{lll}
\hline Localization primary tumor & 263 & $43 \%$ \\
Colon & 474 & $77 \%$ \\
Rectum & 138 & $23 \%$ \\
\hline
\end{tabular}

Staging procedure

Abdominal CT preceding treatment $\quad 513 \quad 84 \%$

Abdominal $\mathrm{CT}<3$ months after surgery $\quad 44 \quad 7 \%$

AJCC stage based on PTNM (2002) ${ }^{a}$

\begin{tabular}{lll} 
Stage 0 & 12 & $2 \%$ \\
Stage I & 75 & $12 \%$ \\
Stage II & 171 & $28 \%$ \\
Stage III & 143 & $23 \%$ \\
Stage IV $^{\text {Not classified }}{ }^{\text {d }}$ & 188 & $31 \%$ \\
\hline
\end{tabular}

Localizations of distant metastases in cohort $^{b}$

Liver $\quad 101 \quad 17 \%$

Peritoneal

Liver and lung

Liver and peritoneal

Lung

Peritoneal and lung

More than 2 organs and/or other localisations ${ }^{c}$

Localization of distant metastases per organ ${ }^{b, c}$

$\begin{array}{lll}\text { Liver } & 144 & 24 \% \\ \text { Peritoneal } & 49 & 8 \% \\ \text { Lung } & 42 & 7 \%\end{array}$

${ }^{a}$ This includes patients with pathological downstaging after neoadjuvant chemoradiation $(n=69)$; a complete remission of histologically proven colorectal cancer was staged as stage 0.

${ }^{b}$ Staging of the lung in this cohort was done with chest CT $(n=415)$ or chest X-ray $(n=197)$.

' Other localisations were brain and skeletal metastases.

d Of 23 patients, 20 patients had no surgery and 3 patients had no resection resulting in an unknown TN status. Of 23 patients, 11 patients had an unknown M status and 12 patients had M0 status.

indeterminate liver lesions were further evaluated with additional diagnostic testing, which were contrastenhanced ultrasound, positron emission tomography (PET), PET/CT or MRI. In 12 patients the indeterminate lesions were diagnosed as metastases and in 7 patients as benign lesions; of these, 6 patients had a follow-up of more then one year including imaging of the liver, and none was diagnosed with metastases. In one patient the liver metastasis was not seen nor suspected on CT, but found during surgery. The ability of the staging $\mathrm{CT}$ to detect liver metastases, summating diagnosis
Table 2 Detection advanced CRC on abdominal CT

\begin{tabular}{|c|c|c|c|c|c|c|c|c|}
\hline \multicolumn{9}{|c|}{ Analysis of surgical patients that were staged before treatment $(n=463)$} \\
\hline \multirow[b]{2}{*}{ Liver metastases } & \multirow{2}{*}{\multicolumn{2}{|c|}{$\mathrm{n}=86$}} & \multicolumn{2}{|c|}{ Diagnosed } & \multicolumn{2}{|c|}{ Suspected } & \multicolumn{2}{|c|}{$\begin{array}{l}\text { Not } \\
\text { seen }\end{array}$} \\
\hline & & & 73 & $85 \%$ & 12 & $14 \%$ & 1 & $1 \%$ \\
\hline $\begin{array}{l}\text { Peritoneal } \\
\text { carcinomatosis }\end{array}$ & & $=33$ & 11 & $33 \%$ & 6 & $18 \%$ & 16 & $48 \%$ \\
\hline \multirow[t]{4}{*}{ pT4 colon carcinoma ${ }^{a}$} & & $=115$ & 99 & $86 \%$ & 5 & $4 \%$ & 11 & $10 \%$ \\
\hline & \multicolumn{4}{|c|}{ Rectum $(n=115)$} & \multicolumn{4}{|c|}{ Colon $(n=348)$} \\
\hline & \multicolumn{2}{|c|}{$\mathrm{cM}$} & \multicolumn{2}{|c|}{$\mathrm{pM}$} & \multicolumn{2}{|c|}{$\mathrm{cM}$} & \multicolumn{2}{|c|}{$\mathrm{pM}$} \\
\hline & $\mathrm{n}$ & $\%$ & $n$ & $\%$ & $n$ & $\%$ & $n$ & $\%$ \\
\hline Liver metastases & 13 & $11 \%$ & 16 & $14 \%$ & 61 & $18 \%$ & 70 & $20 \%$ \\
\hline $\begin{array}{l}\text { Peritoneal } \\
\text { carcinomatosis }\end{array}$ & 2 & $2 \%$ & 3 & $3 \%$ & 9 & $3 \%$ & 30 & $9 \%$ \\
\hline $\begin{array}{l}\text { Locally advanced } \\
\text { disease }\end{array}$ & & 1.a. ${ }^{b}$ & & i.a. & 99 & $29 \%$ & 123 & $36 \%$ \\
\hline
\end{tabular}

CM: metastases diagnosed before treatment on staging abdominal CT (without additional diagnostics)

$\mathrm{pM}$ : final conclusion, including additional imaging, peroperative findings and histology

${ }^{a}$ In 8 patients the local invasiness was not described in the original report, leaving 115 patients for evaluation

${ }^{\mathrm{b}}$ Local invasiveness and lymph node status (CTN stage) in rectal carcinoma was determined on pelvic MRI

on CT and with additional imaging for indeterminate lesions, was 99\%. Peritoneal carcinomatosis was diagnosed in 33 patients; in 11 patients prior to surgery on the staging CT (33\%), in the remaining 22 patients these were found during surgery for the primary tumor. LACC was correctly suggested in 99 out of 115 patients (86\%).

In surgical patients with LACC $(\mathrm{n}=160)$, the incidence of $\mathrm{mCRC}$ was $45 \%(\mathrm{n}=73)$; PC was present in 38 patients (24\%) and LM in 46 patients (29\%). Of the 41 surgical patients with PC, 38 patients had LACC (93\%).

\section{Emergency presentation in surgical patients}

In surgical patients with an emergency presentation $(\mathrm{n}=$ $115)$, the incidence of mCRC was $51 \%(n=59)$ versus $20 \%$ in the elective group $(88 / 436)$. In the same group, limited to colon cancer patients $(\mathrm{n}=110)$, the incidence of LACC was $69 \%(n=76)$ versus $26 \%$ in the elective group (84 out of 317). Staging was omitted in 35 out of 115 patients with an emergency presentation $(30 \%)$, versus 53 out of 436 elective surgical patients (12\%).

\section{Discussion and Conclusions}

Advanced CRC is common, occurring in $58 \%$ of CRC patients in the study. It raises little question at the present that staging before treatment is required. It is presumed the abdominal CT can accurately identify the patients with advanced CRC. For liver metastases and LACC the abdominal CT indeed is an adequate firstline imaging technique, however for PC it grossly lacks 
sensitivity. Appreciating the relationship between LACC and PC, LACC may serve as a warning sign for the possible presence of PC. The incidence of advanced CRC was especially high in patients with emergency presentations, while staging tended to be omitted more often in this group.

The present study was done in a single hospital and therefore has a limited number of patients as compared to true population-based studies. The additive value of this analyses is the prospective design, that is inherently more accurate and provides more detailed clinical data. The outcomes are expected to represent population based incidences fairly good, due to the fact the study was done in an relatively isolated region in the Netherlands that knows very little referral or selection bias. In comparison to a large populationbased study from the Netherlands [19] this study reports a higher incidence of mCRC (colon cancer 34\% vs $25 \%$ and rectal cancer $22 \%$ vs $24 \%$ ). This variance in incidence of $\mathrm{mCRC}$ probably has several causes; such as due to differences in staging routines, mode of data registration, CT scanning protocols and the adverse regional socio-economic status.

Considering potentially curable $\mathrm{mCRC}$, the relevance and outcomes of staging before treatment is most wellknown for liver metastases. Staging with CT before treatment provides the opportunity to change the treatment strategy; various studies have reported promising results of alternative approaches in terms of eligibility of resection and the oncological outcome $[10,11,20]$. Previous reports show a high accuracy of CT for liver metastases, with an initial sensitivity of approximately $85 \%$ which is similar in this study [21]. One study concluded that also small and indeterminate liver lesions should be reported, to optimize the detection rates of true metastases[22]. Our observations agree with that conclusion; Indeterminate lesions in the liver were not a major concern and discrimination of indeterminate liver lesions with additional imaging caused no major diagnostic uncertainties or resources. Contrast-enhanced abdominal MRI is an equivalent alternative to multislice CT scanning for LM, [23] however may cause more logistic problems.

Concerning PC, the benefits of staging before treatment are much less outspoken. In the recent past PC was regarded as a virtually incurable condition with little treatment options. Therefore accurate staging of PC was considered less important. This has changed since the introduction of HIPEC offering a chance for cure in selected patients $[6,12,13]$. Since HIPEC is performed in specialized centers only, accurate pre-operative staging of PC has become vital to improve the outcome of these patients. The present study confirms the finding of previous studies that $\mathrm{PC}$ is poorly visualized on a $\mathrm{CT}$ scan
[24]. Other imaging techniques may be more sensitive to detect and estimate the extent of PC, such as diffusion-weighted imaging (DWI) combined with MRI [25]. This however remains to be proven for its clinical value in colorectal cancer. An alternate approach may be to utilize the observed close relationship between PC and LACC, e.g. by performing a diagnostic laparoscopy in patients with a cT4 tumor on CT; estimation of T stage on abdominal CT in this and another study [26] seems to be fairly reliable.

Standardized radiology reports on $\mathrm{T}$ stage in colon cancer and on localization, sizes, spread and preferably 'weighed' suspicion of distant metastases may enhance the ability to find advanced CRC with the staging abdominal CT.

\section{Authors approval}

All authors have approved on the final version of this manuscript

\section{Acknowledgements}

To M. Elferink en S. Siesling, Comprehensive Cancer Center North-East, for identification of CRC patients that had no surgery.

Funding

This study was performed without funding from grants or (industrial) sponsors.

\section{Author details}

'Department of Surgery, University Medical Center Groningen, (Hanzeplein 1), Groningen, (9713 GZ), the Netherlands. 'Department of Surgery, Medical Spectrum Twente, (Haaksbergerstraat 55), Enschede (7513 ER), the Netherlands. ${ }^{3}$ Department of Radiology, Medical Spectrum Twente, (Haaksbergerstraat 55), Enschede, (7513 ER), the Netherlands. ${ }^{4}$ Department of Surgery, Catharina Hospital, (Michelangelolaan 2), Eindhoven, (5623 EJ), the Netherlands.

\section{Authors' contributions}

TW, IG and JK were responsible for the study design with TW as the principal investigator. IG, JK and WB were responsible for the clinical implementation of staging before treatment with $C T$, data acquisition and critical review of the manuscript. JA was responsible for the clinical implementation of staging with the abdominal $\mathrm{CT}$ at the radiology department, designing the actual scanning protocol, and he functioned as consultant on findings and (additional) imaging techniques. IdH was involved as expert on peritoneal carcinomatosis as co-writer of the manuscript, and functioned as critical reviewer. IG was the study coordinator (at the time of the study she worked in the Medical Spectrum Twente as a resident and the UMCG as a researcher) and wrote the manuscript.

\section{Authors information}

IG: Surgeon, fellow in oncological gastrointestinal surgery. From february 2010 till july 2011 she worked as a fellow at the Catharina Hospital and starting from July 2011 she continues her fellowship at the Medical Spectrum Twente (MST). Further she holds a position as researcher at the UMCG. She previously (also) worked at the MST as a resident in general surgery. This study was part of her thesis "Searching for metastases in colorectal cancer" (University of Groningen).

$J K$ : Oncological gastrointestinal surgeon, specialized in gastrointestinal and hepatobiliary surgery. Tutor for scientific research at the Medical Spectrum Twente.

JA: Staff radiologist at the Medical Spectrum Twente

$\mathrm{IdH}$ : Oncological gastrointestinal surgeon, specialized in treatment of peritoneal carcinomatosis (PC) and head of the research group on PC at the Catharina Hospital. 
WM: Oncological and gastrointestinal surgeon, specialized in colorectal and head/neck surgery. Responsible tutor of the trainees in general surgery at the Medical Spectrum Twente.

TW: Professor in oncological surgery and former head of department at the UMC Groningen.

\section{Competing interests}

The authors declare that they have no competing interests.

Received: 15 May 2011 Accepted: 7 October 2011

Published: 7 October 2011

\section{References}

1. Povoski SP, Fong Y, Sgouros SC, Kemeny NE, Downey RJ, Blumgart LH: Role of chest $\mathrm{CT}$ in patients with negative chest x-rays referred for hepatic colorectal metastases. Ann Surg Oncol 1998, 5:9-15.

2. Brent A, Talbot R, Coyne J, Nash G: Should indeterminate lung lesions reported on staging $C T$ scans influence the management of patients with colorectal cancer? Colorectal Dis 2007, 9:816-818.

3. Grossmann I, Avenarius JK, Mastboom WJ, Klaase JM: Preoperative staging with chest CT in patients with colorectal carcinoma: Not as a routine procedure. Ann Surg Oncol 2010, 17:2045-2050.

4. Adam R, Hoti E, Folprecht G, Benson AB: Accomplishments in 2008 in the management of curable metastatic colorectal cancer. Gastrointest Cancer Res 2009, 3(Suppl):15-22.

5. Bentrem DJ, DeMatteo RP, Blumgart LH: Surgical therapy for metastatic disease to the liver. Annu Rev Med 2005, 56:139-156.

6. Cao C, Yan TD, Black D, Morris DL: A systematic review and meta-analysis of cytoreductive surgery with perioperative intraperitoneal chemotherapy for peritoneal carcinomatosis of colorectal origin. Ann Surg Oncol 2009, 16:2152-2165.

7. Elferink MA, van Steenbergen LN, Krijnen P, Lemmens VE, Rutten HJT, Marijnen CA, et al: Marked improvements in survival of patients with rectal cancer in the netherlands following changes in therapy, 19892006. Eur J Cancer 2010, 46:1421-9.

8. Elias D, Liberale G, Vernerey D, Pocard M, Ducreux M, Boige V, et al: Hepatic and extrahepatic colorectal metastases: When resectable, their localization does not matter, but their total number has a prognostic effect. Ann Surg Oncol 2005, 12:900-909.

9. Neeff H, Hörth W, Makowiec F, Fischer E, Imdahl A, Hopt UT, Passlick B: Outcome after resection of hepatic and pulmonary metastases of colorectal cancer. J Gastrointest Surg 2009, 13:1813-1820.

10. van der Pool AE, de Wilt JH, Lalmahomed ZS, Eggermont AM, ljzermans JN, Verhoef $C$ : Optimizing the outcome of surgery in patients with rectal cancer and synchronous liver metastases. Br J Surg 2010, 97:383-390.

11. Reddy SK, Barbas AS, Clary BM: Synchronous colorectal liver metastases: Is it time to reconsider traditional paradigms of management? Ann Surg Oncol 2009, 16:2395-2410.

12. Sugarbaker $\mathrm{PH}$ : A curative approach to peritoneal carcinomatosis from colorectal cancer. Semin Oncol 2005, 32(Suppl):68-73.

13. Verwaal VJ: Long-Term results of cytoreduction and HIPEC followed by systemic chemotherapy. Cancer J 2009, 15:212-5.

14. Carmignani $\mathrm{CP}$, Ortega-Perez G, Sugarbaker PH: The management of synchronous peritoneal carcinomatosis and hematogenous metastasis from colorectal cancer. Eur J Surg Oncol 2004, 30:391-398.

15. van Steenbergen LN, Elferink MA, Krijnen P, Lemmens VE, Siesling $S$, Rutten $\mathrm{HJ}$, et al: Improved survival of colon cancer due to improved treatment and detection: A nationwide population-based study in the Netherlands 1989-2006. Ann Oncol 2010, 21:2206-2212.

16. Karoui M, Soprani A, Charachon A, Delbaldo C, Vigano L, Luciani A, Cherqui D: Primary chemotherapy with or without colonic stent for management of irresectable stage IV colorectal cancer. Eur J Surg Oncol 2010, 36:58-64.

17. Poultsides GA, Servais EL, Saltz LB, Patil S, Kemeny NE, Guillem JG, et al: Outcome of primary tumor in patients with synchronous stage IV colorectal cancer receiving combination chemotherapy without surgery as initial treatment. J Clin Oncol 2009, 27:3379-3384.

18. Scoggins CR, Meszoely IM, Blanke CD, Beauchamp RD, Leach SD: Nonoperative management of primary colorectal cancer in patients with stage IV disease. Ann Surg Oncol 1999, 6:651-657.
19. Lemmens $V$, van Steenbergen $L$, Janssen-Heijnen Maryska, Martijn $H$ Rutten HJT, Coebergh JW: Trends in colorectal cancer in the south of the Netherlands 1975-2007: Rectal cancer survival levels with colon cancer survival. Acta Oncologica 2010, 49:784-796.

20. Adam R, Vinet E: Regional treatment of metastasis: Surgery of colorectal liver metastases. Ann Oncol 2004, 15(Suppl 4):103-106.

21. Valls C, Andía E, Sánchez A, Gumà A, Figueras J, Torras J, Serrano T: Hepatic metastases from colorectal cancer: preoperative detection and assessment of resectability with helical CT. Radiology 2001, 218:55-60.

22. van Erkel AR, Pijl MEJ, van den Berg-Huysmans AA, Wasser MNJM, van de Velde CJH, Bloem JL: Hepatic metastases in patients with colorectal cancer: Relationship between size of metastases, standard of reference, and detection rates. Radiology 2002, 224:404-409.

23. van Etten B, Van der Sijp JRM, Kruyt RH, Oudkerk M, Van der Holt B, Wiggers T: Ferumoxide-Enhanced magnetic resonance imaging techniques in pre-operative assessment for colorectal liver metastases. Eur J Surg Oncol 2002, 28:645-651.

24. de Bree E, Koops W, Kröger R, van Ruth S, Verwaal VJ, Zoetmulder FA: Preoperative computed tomography and selection of patients with colorectal peritoneal carcinomatosis for cytoreductive surgery and hyperthermic intraperitoneal chemotherapy. Eur J Surg Oncol 2006, 32:65-71.

25. Low RN, Sebrechts CP, Barone RM, Muller W: Diffusion-Weighted MRI of peritoneal tumors: Comparison with conventional MRI and surgical and histopathologic findings-a feasibility study. Am J Roentgenol 2009, 193:461-470.

26. Kanamoto T, Matsuki M, Okuda J, Inada Y, Tatsugami F, Tanikake M, et al: Preoperative evaluation of local invasion and metastatic lymph nodes of colorectal cancer and mesenteric vascular variations using multidetector-row computed tomography before laparoscopic surgery. J Comput Assist Tomogr 2007, 31:831-839.

\section{Pre-publication history}

The pre-publication history for this paper can be accessed here: http://www.biomedcentral.com/1471-2407/11/433/prepub

doi:10.1186/1471-2407-11-433

Cite this article as: Grossmann et al:: The strengths and limitations of routine staging before treatment with abdominal $\mathrm{CT}$ in colorectal cancer. BMC Cancer 2011 11:433.

\section{Submit your next manuscript to BioMed Central and take full advantage of:}

- Convenient online submission

- Thorough peer review

- No space constraints or color figure charges

- Immediate publication on acceptance

- Inclusion in PubMed, CAS, Scopus and Google Scholar

- Research which is freely available for redistribution

Submit your manuscript at www.biomedcentral.com/submit
C Biomed Central 